\title{
Recurrent structural variations of IncRNA gene CCDC26 in diffuse intrinsic pontine glioma
}

Lihua Zou (lihuazou@gmail.com)

Northwestern University

Chicago, IL, USA

\section{Abstract}

We report recurrent somatic structural variations (SVs) involving long noncoding RNA (IncRNA) CCDC26 in 13\% of Diffuse Intrinsic Pontine Glioma (DIPG) patients. We validate our findings using whole genome sequencing data from two independent patient cohorts. CCDC26 SVs cause increased expression of $C C D C 26$ gene in patients. In addition, CCDC26 expression is associated with elevated expression of $M Y C$ and proliferation signature. Our findings identify CCDC26 as a novel significantly mutated gene in DIPG and highlight the importance of structural variations in pediatric brain cancer.

\section{Main}

Diffuse intrinsic pontine glioma (DIPG), the most frequent brainstem tumor in pediatric patients, is one of the most devastating childhood cancers, and virtually all DIPG patients die within two years after diagnosis. Current standard of care, chemotherapy followed by radiation, yields no improvements in survival. There is an unmet need for the identification of molecular mechanisms and efficacious therapeutic agents to improve treatment outcomes for DIPG patients. The discovery of somatic histone gene mutations, resulting in replacement of lysine 27 by methionine (K27M) in the encoded histone $\mathrm{H} 3$ proteins, in DIPG has dramatically improved our understanding of disease pathogenesis and stimulated the development of novel therapeutic approaches for the treatment of DIPG ${ }^{1,2}$. Past sequencing analyses of DIPG were largely focused on somatic point mutation or chromosome copy 
number alteration ${ }^{3}$. Structural variation (SV) is another class of mutations that can lead to duplication, deletion or reordering of DNA at scales ranging from single genes to entire chromosomes. The role of SVs in DIPG is poorly understood. To address this issue, we analyzed the whole genome sequences of matched tumor and normal pairs from 60 DIPG patients. These patients were from two independent cohorts: one from the CBTTC OpenDIPG project $(n=45)$ and a second from PNOS project $(n=15)$ (Methods). These data enable a gene-centric approach to detect SVs in DIPG.

Strikingly, we discovered recurrent SV mutations at IncRNA CCDC26 in 13\% of DIPG patients (8 out of 60) from the combined cohorts (Fig.1a). The role of CCDC26 in cancer is not well understood. It was implicated in childhood acute myeloid leukemia (AML) because of altered chromosome copy numbers in AML patients ${ }^{4}$. We examined 8 patients with recurrent CCDC26 SVs sample by sample. We found 6 out of 8 patients have CCDC26 amplified through tandem duplication. To delineate critical functional region of CCDC26, we overlaid sequences altered by SVs at CCDC26 locus and pinpointed a common 140kb amplicon at chr8:129,523,594-129,662,989 on hg38 reference genome (Fig.1b). Notably, the common amplicon is next to a germline SNP (rs4295627) associated with 1.3-fold increased risk in glioma development ${ }^{5}$. We observed three samples have tandem duplication breakpoints intersecting with the exon region of two neighboring genes GSDMC and FAM49B (Fig.1b). We searched for gene fusions involving CCDC26 and GSDMC or FAM49B using RNA-Seq from matched samples. In patient BS_1Q524P3B, we observed a gene fusion joining transcripts from CCDC26 exon 1 and GSDMC exon 7-14 (Fig.1c). GSDMC encodes Gasdermin-C, a protein coding gene which may be acting by homooligomerizing within the membrane and forming pores. We observed two patients (BS_CBMAWSAR and BS_FKQ7F6D1) displaying highly amplified DNA segments involving many breakpoints (>=100) proximal to CCDC26. The high copy numbers changes linked across multiple distant chromosome segments suggest ecDNA (e.g. double minute, neochromosome) as underlying structure (Fig.1e; Fig.2c) ${ }^{6-8}$. ecDNA has been reported as a 
mechanism which can lead to amplification of driver oncogene under selection pressure, ${ }^{8}$. Consistent with this, we observed co-amplified breakpoints involving CCDC26 on chromosome 8 and EGFR on chromosome 7 In patient BS_CBMAWSAR (Fig.1f). We also observed co-amplified breakpoints involving CCDC26 and MYC on chromosome 8 in patient BS_FKQ7F6D1 (Fig.2d).

To study the functional impact of CCDC26 SVs, we compared CCDC26 expression between the samples in the presence and absence of SVs (CCDC26-SV vs. CCDC26-WT) using RNA-Seq from matched samples. CCDC26-SV samples have significantly higher CCDC26 expression than CCDC26-WT samples (Fig.2a; t-test $p<0.05)$ indicating a functional role of CCDC26 in DIPG. To identify differential genes and pathways between CCDC26-SV and CCDC26-WT, we performed differential gene expression analysis using DESeq $2^{9}$ and found MYC up-regulated in CCDC26-SV samples $(p<0.005)$. We performed Gene Set Enrichment Analysis $(G S E A)^{10}$ and found proliferation signatures and MYC targets enriched in CCDC26-SV samples (FDR q<0.1). In addition, CCDC26 expression is correlated with MYC expression in our cohorts (Fig.2b; Pearson correlation p<0.005).

Taken together, our findings identify CCDC26 as a frequently mutated IncRNA gene in DIPG. Expression analysis suggests CCDC26 is associated with MYC and proliferation pathways.The detailed molecular mechanism of CCDC26 remains to be elucidated. Our findings highlight the importance of studying genome structural rearrangements in this deadly disease. 


\section{Methods}

\section{Cohort description}

The 60 DIPG specimens used in our study are composed of radiologically diagnosed DIPG from Children's Brain Tumor Tissue Consortium (CBTTC) and the Pediatric Pacific Neuro-oncology Consortium (PNOC). The raw whole genome sequencing and RNA-seq data can be downloaded from the Gabriella Miller Kids First Data Resource Center (KF-DRC). The CBTTC is a collaborative, multi-institutional research program dedicated to the study of childhood brain tumors. The Pacific Pediatric Neuro-Oncology Consortium (PNOC) is an international consortium dedicated to bringing new therapies to children and young adults with brain tumors. PNOC collected blood and tumor biospecimens from newly diagnosed DIPG patients as part of the clinical trial PNOC003/NCT02274987.

\section{Whole-genome sequencing analysis}

Paired-end DNA-Seq reads were aligned to hg38 (patch release 12) reference genome using BWA-MEM ${ }^{11}$. Duplicates were marked using Samblaster ${ }^{12}$. BAMs were merged and processed using Broad's Genome Analysis Toolkit (GATK) ${ }^{13}$. For WGS variant calling, Strelka2 ${ }^{14}$ was used to call Indels and Mutect ${ }^{15}$ was used to call SNVs using default parameters. The final Strelka2 and Mutect2 VCFs were filtered for PASS variants for downstream analysis. For structural variant (SV) calls, Manta $^{16}$ was used using hg38 as reference genome. Manta SV output was annotated using AnnotSV ${ }^{17}$. The docker image of Whole Genome Sequence Analysis Workflow can be found in the KidsFirst GitHub repository.

\section{Gene expression analysis}

Paired-end RNA-Seq reads were aligned using ENSEMBL's GENCODE 27 as the reference genome. Transcript- and gene-level expression values were calculated using RSEM ${ }^{18}$. Data normalization and 
differential gene expression were done by DESeq $2^{9}$. Gene set enrichment analysis $(\text { GSEA })^{10}$ was used to find groups of enriched genes between different groups of samples.

\section{Gene fusion analysis}

Gene fusions were called using Arriba ${ }^{19}$. Gene fusion calls from Arriba were annotated using FusionAnnotator (https://github.com/FusionAnnotator) followed by filtering of recurrent fusion artifacts and transcripts present in normal tissue using a blacklist file bundled with Arriba.

\section{Figures and Legends}

Figure 1. Recurrent structural variants of CCDC26 in DIPG a) Gene-centric table showing frequency of CCDC26 SVs along with previously reported driver mutations in DIPG in the combined cohorts; b) Common CCDC26 amplicon (indicated by grey bar in the top panel) altered by SVs; supporting reads at breakpoints of CCDC26 and GSDMC are shown in the bottom panel; c) SV and copy number changes associated with CCDC26-GSDMC in patient BS_1Q524P3B; d) Structure of gene fusion CCDC26-GSDMC identified in RNA-Seq of patient BS_1Q524P3B; e) Circos plots showing clustering of breakpoints at chromosome 7 and 8 in patient BS_CBMAWSAR; f) SVs (top panel) and associated high copy number changes (bottom panel) on chromosome 7 and 8 in patient BS_CBMAWSAR. Colored curves in the top panel encode different types of SVs (red: tandem duplication; blue: deletion; green: 5'Inversion; orange: 3’Inversion; purple: translocation).

Figure 2. Impact of CCDC26 SVs on gene expression. a) Expression of CCDC26 in CCDC26-SV vs. CCDC26-WT; y-axis indicate log2-transformed RSEM gene expression value measured by RNA-Seq; b) Correlation between CCDC26 and MYC expression (Pearson correlation; p<0.005); $x$-axis and $y$-axis indicate log2-transformed RSEM gene expression value measured by RNA-Seq; c) Circos plots showing clustering of breakpoints on chromosome 8 in patient BS_FKQ7FD1; d) Detailed 
view of SV breakpoints and high copy number changes involving CCDC26 and MYC on chromosome 8 in patient BS_FKQ7FD1.

\section{References}

1. Schwartzentruber, J. et al. Driver mutations in histone H3.3 and chromatin remodelling genes in paediatric glioblastoma. Nature 482, 226-231 (2012).

2. Wu, G. et al. Somatic histone $\mathrm{H} 3$ alterations in pediatric diffuse intrinsic pontine gliomas and non-brainstem glioblastomas. Nat. Genet. 44, 251-253 (2012).

3. Mackay, A. et al. Integrated Molecular Meta-Analysis of 1,000 Pediatric High-Grade and Diffuse Intrinsic Pontine Glioma. Cancer Cell 32, 520-537.e5 (2017).

4. Storlazzi, C. T. et al. Identification of a commonly amplified 4.3 Mb region with overexpression of C8FW, but not MYC in MYC-containing double minutes in myeloid malignancies. Hum. Mol. Genet. 13, 1479-1485 (2004).

5. Shete, S. et al. Genome-wide association study identifies five susceptibility loci for glioma. Nat. Genet. 41, 899-904 (2009).

6. Zhang, C.-Z. et al. Chromothripsis from DNA damage in micronuclei. Nature 522, 179-184 (2015).

7. Turner, K. M. et al. Extrachromosomal oncogene amplification drives tumour evolution and genetic heterogeneity. Nature vol. 543 122-125 (2017).

8. Garsed, D. W. et al. The architecture and evolution of cancer neochromosomes. Cancer Cell 26, 653-667 (2014).

9. Love, M. I. et al. Moderated estimation of fold change and dispersion for RNA-seq data with DESeq2. Genome Biol. 15, 550 (2014).

10. Subramanian, A. et al. Gene set enrichment analysis: a knowledge-based approach for 
interpreting genome-wide expression profiles. Proc. Natl. Acad. Sci. U. S. A. 102, 15545-15550 (2005).

11. Li, H. \& Durbin, R. Fast and accurate long-read alignment with Burrows-Wheeler transform. Bioinformatics 26, 589-595 (2010).

12. Faust, G. G. \& Hall, I. M. SAMBLASTER: fast duplicate marking and structural variant read extraction. Bioinformatics 30, 2503-2505 (2014).

13. McKenna, A. et al. The Genome Analysis Toolkit: a MapReduce framework for analyzing next-generation DNA sequencing data. Genome Res. 20, 1297-1303 (2010).

14. Saunders, C. T., Wong, W. S. W., Swamy, S. \& Becq, J. Strelka: accurate somatic small-variant calling from sequenced tumor-normal sample pairs. (2012).

15. Cibulskis, K. et al. Sensitive detection of somatic point mutations in impure and heterogeneous cancer samples. Nat. Biotechnol. 31, 213-219 (2013).

16. Chen, X. et al. Manta: rapid detection of structural variants and indels for germline and cancer sequencing applications. Bioinformatics 32, 1220-1222 (2016).

17. Geoffroy, V. et al. AnnotSV: an integrated tool for structural variations annotation. Bioinformatics 34, 3572-3574 (2018).

18. Li, B. \& Dewey, C. N. RSEM: accurate transcript quantification from RNA-Seq data with or without a reference genome. BMC Bioinformatics 12, 323 (2011).

19. Uhrig, S., Fröhlich, M., Hutter, B. \& Brors, B. PO-400 Arriba - fast and accurate gene fusion detection from rna-seq data. Epigenetic Mechanisms (2018) doi:10.1136/esmoopen-2018-eacr25.427.

\section{Acknowledgements}

We thank members of Children's Brain Tumor Tissue Consortium (CBTTC) (www.cbttc.org) for their support of open access, biospecimen driven research. 
a

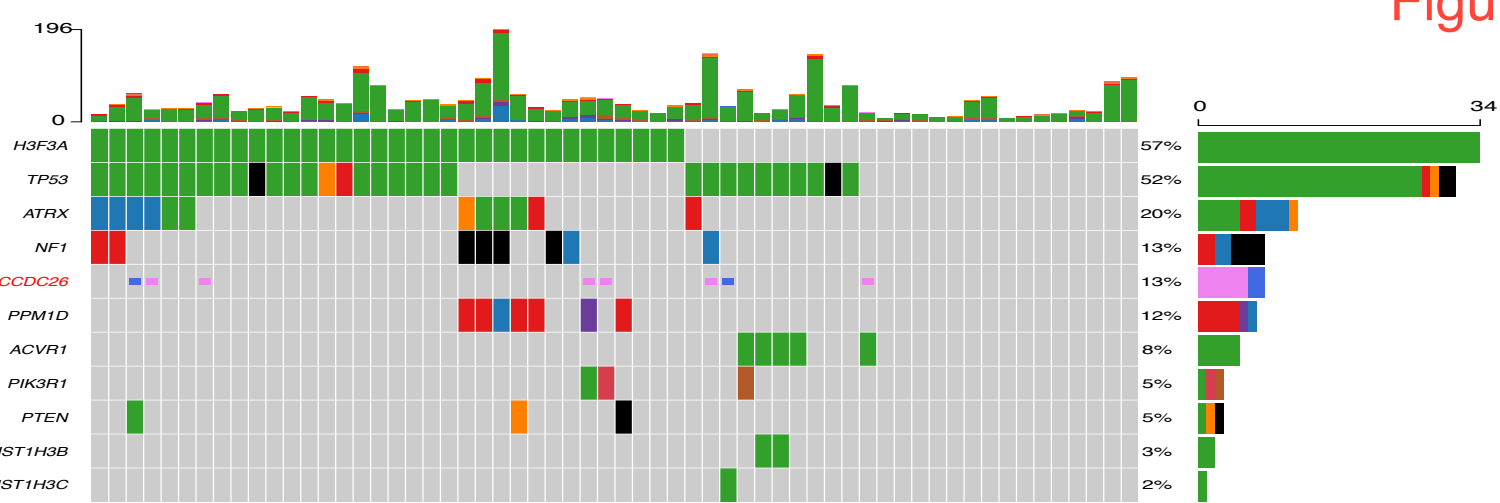

- Missense_Mutation - In_Frame_Ins - Frame_Shift_Ins \& Splice_Site " Tandem Dup

- Nonsense_Mutation - In_Frame_Del - Frame_Shift_Del - Multi_Hit - Double Minute

b
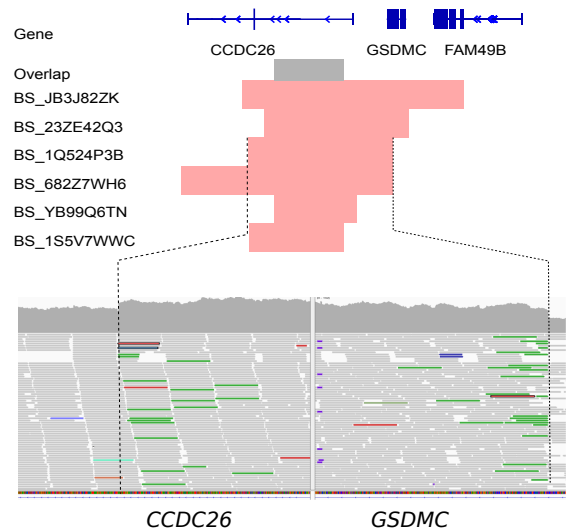

e
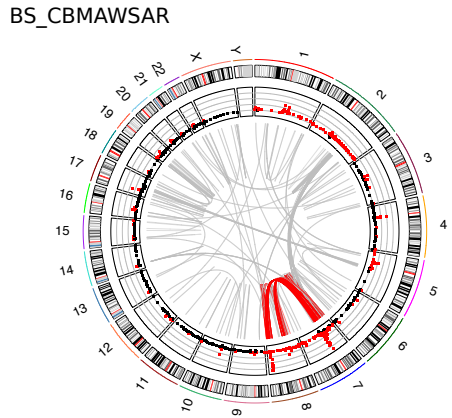

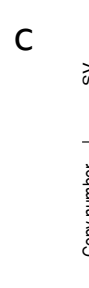

BS 1 Q 2524 P3B

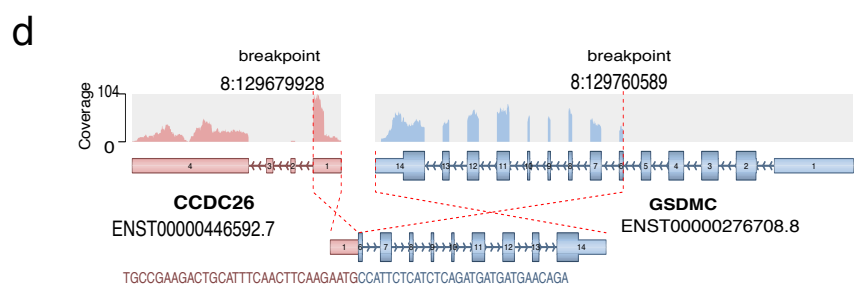

f

BS_CBMAWSAR

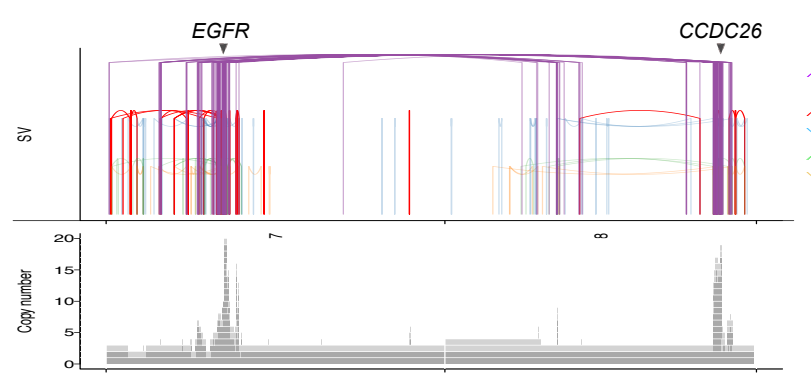



a

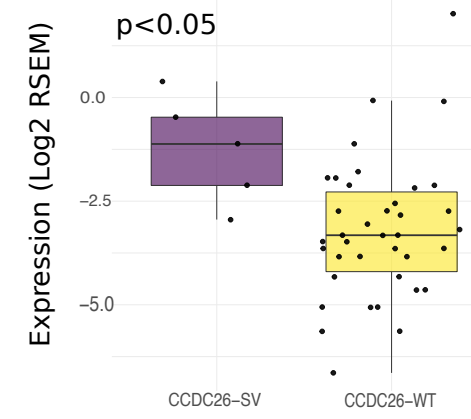

\section{BS_FKQ7F6D1}

C

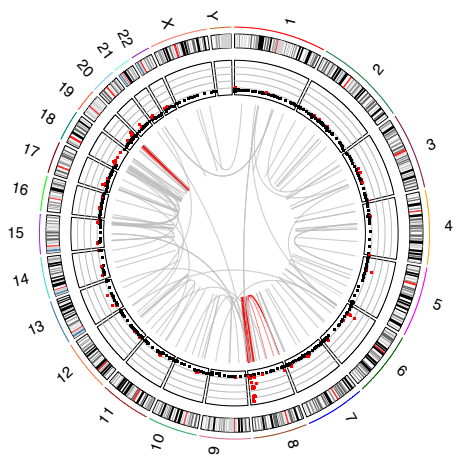

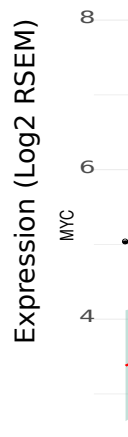

$p<0.005$

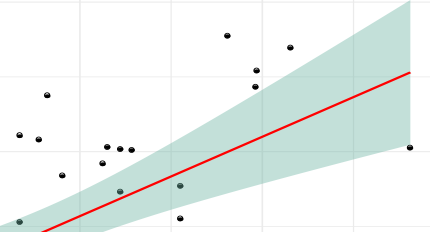

Figure 2 d

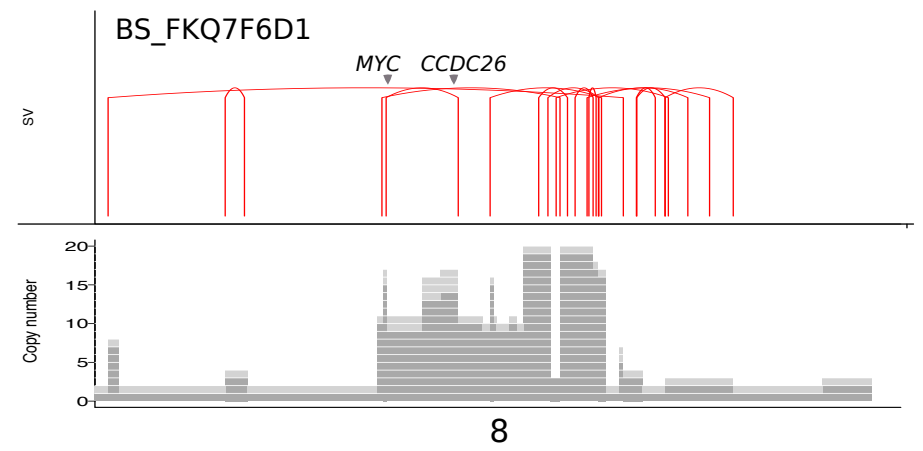

